\title{
Reply to the comment by I. R. Mann and G. Chisham
}

\author{
K.-H. Glassmeier \\ Institute for Geophysics and Meteorology, Technical University of Braunschweig, Germany
}

Received: 23 August 1999 / Accepted: 5 October 1999

\section{General comments}

In their comment on the paper by Glassmeier et al. (1999), Mann and Chisham (1999) criticise the purely mathematical analysis used to derive a non-integer drift-bounce resonance condition in asymmetric magnetospheric ULF pulsation wave fields. They claim that Glassmeier et al.'s (1999) treatment is flawed because of an incorrect treatment of the ULF pulsation wave electric field's variation along the background magnetic field. Furthermore, Mann and Chisham (1999) consider that Glassmeier et al. (1999) miss some important aspect of the physics of the process, that is they do not consider the initial particle bounce phase. Mann and Chisham (1999) claim that "when the correct analysis is undertaken it becomes clear that $N$ must be an integer for a genuine resonance to occur". However, nowhere in their comment do they present this "correct analysis". In reply to their critism I would like to note that Mann and Chisham (1999) do not present a detailed mathematical analysis and correction of the process under consideration, as would be appropriate. The graphical approach used is not at all satisfactory to use as an argument against the mathematical analysis presented by Glassmeier et al. (1999). Figures 1-4 of Mann and Chisham (1999) display the wave electric field as a function of the azimuthal and magnetic field-aligned coordinates at a specific instance of time, while the particle trajectory is displayed for many occasions. This approach might be useful for illustrative purposes, but does not allow a detailed study of the process at all. The mathematical analysis presented by Glassmeier et al. (1999) shows that maximum energy transfer from the particles to the wave occurs in a resonant way, i.e. at resonance the particles always "see" or experience the same electric field. This basic resonance property is not reflected in Mann and Chisham's (1999) graphical approach at all. Furthermore, phase considerations are not as important as claimed by Mann and Chisham (1999) as the electric field is constant in the frame of reference of the resonant particles. The criticism by Mann and Chisham (1999) is thus inappropriate.

\section{Derivation of the generalized resonance condition}

However, the comment indicates that the new result derived by Glassmeier et al. (1999) needs further explanation and clarification. Here, I shall try a slightly different approach to demonstrate the correctness of the mathematical analysis of Glassmeier et al. (1999). This approach parallels the treatment of the ion-cyclotron resonance of Brice (1964). Furthermore, an inconsistency in the definition of the arc length $s$ as used by Glassmeier et al. (1999) is corrected.

The incremental change in energy of a particle, $\delta W$, is given by the scalar product of the force $\vec{F}=q \vec{E}$ and the incremental distance $\delta \vec{s}=\vec{v} \delta t$ (e.g. Brice, 1964):

$\delta W=q \vec{E} \cdot \vec{v} \delta t$.

Here $q$ is the electric charge of the particle, $\vec{E}$ the wave electric field, and $\vec{v}$ the particle velocity.

In the present case Eq. (1) reads (e.g. Glassmeier et al., 1999)

$\delta W_{B}(s)=q E_{\phi}(s) v_{D}(s) \exp (i(m \phi-\omega t)) \delta t$

where $E_{\phi}(s)$ is the arc length $s$ dependent azimuthal electric field component of the wave, $v_{D}(s)$ the arc length-dependent azimuthal drift velocity, and $\phi$ the azimuth angle or azimuthal drift phase. The arc length is counted positive from the northern ionospheric footpoint of the field line. The southern ionospheric footpoint is at $s=L$, where $L$ is the length of the field line. At the equator $s=L / 2$. The electric field may be written as

$E_{\phi}(s)=-i E_{0} \exp \left(i \frac{\alpha \pi}{L} s\right)$

This expression allows for asymmetric electric field variations with respect to the equatorial plane. The 
parameters $\alpha$ describes the asymmetry of the oscillating wave field along the background magnetic field.

This form of the electric field variation along the magnetic field is commonly used in ULF pulsation studies (e.g. Hughes, 1983), and it provides a fair approximation to the variation of the field with $s$. The wave form suggested by Mann and Chisham (1999), $E_{\phi}(s)=E_{0}(s) \exp (i \chi(s))$, is a suitable form if both the amplitude and the phase vary along the field, i.e. if the phase variation is modulated by a slower amplitude variation. If, however, $\chi(s)=0$, as claimed by Mann and Chisham (1999), then all the spatial variation of the electric field is in $E_{0}(s)$. This spatial variation can be approximated by Eq. (3), too. Thus, I cannot see any good reason for critizing Eq. (3).

For a particle bouncing in a dipole field one has:

$s=\frac{L}{2}-\frac{l}{2} \cos \Omega_{B} t$

with $\Omega_{B}$ the bounce frequency of the resonant particles, and $l$ the half-bounce path length, that is the rectified path distance between the northern and southern mirror points of the bouncing particle; at time $t=0$ the particle position is at its northern mirror point $s_{N}=(L-l) / 2$.

Now, the electric field as seen by a bouncing particle is given by:

$E_{\phi}(s) \propto \exp \left(-i \frac{\alpha \pi l}{2 L} \cos \Omega_{B} t\right)$.

Note that Eq. (5) is the corrected version of Eq. (8) of Glassmeier et al. (1999). With $v_{D}(s) \approx\left\langle v_{D}(s)\right\rangle=v_{D}$, and $\phi(t)=\Omega_{D} t$ the incremental energy change of a particle due to its interaction with the electromagnetic field is now given by

$\delta W_{B} \propto E_{0} v_{D} \exp \left(i\left(m \Omega_{D}-\omega\right) t-i \frac{\alpha \pi l}{2 L} \cos \Omega_{B} t\right) \delta t$,

where $\Omega_{D}$ denotes the drift frequency.

Following Brice (1964) I require the scalar product of the wave electric field and the particle velocity, or, equivalently, the incremental energy change $\delta W_{B}$ to have a constant (zero) frequency component to obtain a significant amount of energy transfer. This condition avoids integration of the energy change over a particle bounce period, reflecting the concept that a resonant particle "feels" the same electric field during its bounce motion, and also taking care of Mann and Chisham's (1999) phase argument.

For the case of an ion cyclotron resonance this requirement leads immediately to the well-known cyclotron resonance condition (Brice, 1964). In the ion cyclotron case the gyrophase is linearly related to time $t$. To achieve a similar linear relation for the bounce phase Glassmeier et al. (1999) have introduced a triangular approximation for $s(t)$, which reads, in its corrected version, as

$s(t) \approx\left\{\begin{array}{ll}\frac{L}{2}-\frac{l}{2}+\frac{l}{\pi} \Omega_{B} \cdot t & \text { if } 0 \leq t \leq T_{B} / 2 \\ \frac{L}{2}+\frac{3 l}{2}-\frac{1}{\pi} \Omega_{B} \cdot t & \text { if } T_{B} / 2 \leq t \leq T_{B}\end{array}\right.$,
Thus,

$\delta W_{B} \propto\left\{\begin{array}{l}\exp \left(i\left(m \Omega_{D}-n \Omega_{B}-\omega\right) \cdot t\right) \delta t \quad \text { if } 0 \leq t \leq T_{B} / 2 \\ \exp \left(i\left(m \Omega_{D}+n \Omega_{B}-\omega\right) \cdot t\right) \delta t\end{array}\right.$

where $n=\alpha l / L$ has been introduced. A zero frequency component exists if

$\omega-m \Omega_{D} \pm n \Omega_{B}=0$,

where the ' + ' ('-') sign indicates resonant energy transfer from the "downward" ("upward") moving particles, i.e. the energy increment is resonant for the time interval $0 \leq t \leq T_{B} / 2\left(T_{B} / 2 \leq t \leq T_{B}\right)$. Note, that if the "downward" resonance condition with $+n$ is fulfilled the "upward" condition with $-n$ is not satisfied, which implies that a net energy increment exists. The discrimination between the "downward" and "upward" resonance is somewhat equivalent to ion cyclotron resonances with right and left hand polarized waves.

Equation (9) with $n \in \mathscr{R}$ is a proper generalization of Southwood's (1976) resonance condition. It should be noted that $n$ is an integer if $\alpha=1$ and $l=L$. Thus, the resonance condition derived here includes as a special case the more restricted condition $n \in \mathscr{N}$. Furthermore, $n$ is not an arbitrary number, but reflects physical conditions of the system under consideration. The actual value of $n$ depends on the ionospheric conditions in the Northern and Southern Hemisphere of the magnetosphere as well as the length of the bouncing particle path length.

When comparing ion cyclotron and drift-bounce resonance instability both $l$ and $L$ correspond to the length of the gyrocircumference, that is $2 \pi r_{g}$, where $r_{g}$ is the gyroradius; $\alpha \in \mathscr{N}$ in the ion cyclotron case. Therefore, an analysis as outlined here naturally leads to the well-known resonance condition for the ion cyclotron instability with $n \in \mathscr{N}$. As already noted by Glassmeier et al. (1999) the condition $n \in \mathscr{N}$ for the drift-bounce resonance instability goes back to a very special treatment of the expression $\exp \left(i \frac{\alpha \pi l}{2 L} \cos \Omega_{B} t\right)$ in Eq. (5), which can be interpreted as a generating function for the Bessel functions $J_{N}$ :

$\exp \left(i \frac{\alpha \pi l}{2 L} \cos \Omega_{B} t\right)=\sum_{N=-\infty}^{+\infty} \mathrm{e}^{i N \pi / 2} J_{N}\left(\frac{\alpha \pi l}{2 L}\right) \exp \left(i N \Omega_{B} t\right)$

where $N$ is an integer. It is this series expansion which imposes the $n \in \mathscr{N}$ condition. The triangular approximation introduced by Glassmeier et al. (1999) allows a much more general and physical interpretation of $n$. The number $n$ is a parameter measuring the asymmetry of the electric field along $s$, the length the field line, and the bounce length of the resonating particle.

\section{Conclusion}

Based on an argument by Brice (1964) I have tried to use a somewhat different approach in deriving the generalised drift-bounce resonance condition. The criticism of 
Mann and Chisham (1999) is not justified. The results achieved by Glassmeier et al. (1999) are correct, significant, and allow a far reaching interpretation of geomagnetic giant pulsations.

\section{References}

Brice, N., Fundamentals of very low frequency emission generation mechanisms, J. Geophys. Res., 69, 4515-4522, 1964.

Glassmeier, K. H., S. Buchert, U. Motschmann, A. Korth, and A. Pedersen, Concerning the generation of geomagnetic giant pulsations by drift-bounce resonance ring current instabilities, Ann. Geophysicae, 17, 338-350, 1999.

Hughes, W., Hydromagnetic waves in the magnetosphere, in Eds. R. L. Carovillano, J. M. Forbes, Solar-terrestrial physics, pp 453-477, D. Reidel, Dordrecht, 1983.

Mann, I. R., and G. Chisham, Comment on "Concerning the generation of geomagnetic giant pulsations by drift-bounce resonance ring current instabilities" by K. H. Glassmeier et al., Ann. Geophysicae, 17, 338-350, (1999), Ann. Geophysicae, in press, 1999.

Southwood, D. J., A general approach to low-frequency instability in the ring current plasma, J. Geophys. Res., 81, 3340, 1976. 